\title{
The Choice Should Be Yours: Diabetes-Related Distress by Insulin Delivery Method for People with Type 1 Diabetes
}

\author{
Jana L. Wardian, $\mathrm{PhD}^{1}$ Mark W. True, $M D^{2}$ Irene Folaron, $\mathrm{MD}^{2}$ Jeff Colburn, $\mathrm{MD}^{2}$ \\ Joshua M. Tate, $\mathrm{MD}^{2}$ and Darrick J. Beckman, $\mathrm{MD}^{1}$
}

\begin{abstract}
Background: American Diabetes Association (ADA) recommends psychosocial assessment for people with diabetes, including diabetes-related distress. Elevated diabetes-related distress is associated with poor selfmanagement, lower medication adherence, and poorer quality of life. Insulin delivery methods are multiple daily injections (MDI) or continuous subcutaneous insulin infusion (CSII). Because people with type 1 diabetes mellitus (T1DM) require comprehensive insulin therapy to manage blood glucose, we explored the association of insulin delivery methods and diabetes distress in this group.

Methods: The U.S. Air Force Diabetes Center of Excellence (DCOE), a specialty clinic for adults who are Military Health System beneficiaries, administers the validated 17-item Diabetes-related Distress Scale (DDS17) as part of standard care. Patient data were analyzed from June 2015 to August 2016 using SPSS version 22. Patients were free to choose the method of insulin delivery with minimal or no additional cost.

Results: There were 203 patients with T1DM who completed the DDS-17 as part of standard care during the time period. Patients were categorized as CSII $(57.6 \%)$ or MDI $(42.4 \%)$. Women were significantly more likely to choose MDI over CSII than men $(P=0.003)$. DDS-17 scores were low in both groups, and there were no significant differences in DDS-17 by insulin delivery method. Furthermore, no significant differences were found in hemoglobin A1c (HbAlc) between CSII $(7.9 \%$ or $63 \mathrm{mmol} / \mathrm{mol})$ and MDI $(8.1 \%$ or $65 \mathrm{mmol} / \mathrm{mol})$ users $(P=0.22)$ and no significant differences in body mass index (BMI) between patients using CSII $\left(M=28.33 \mathrm{~kg} / \mathrm{m}^{2}\right)$ and MDI $\left(28.49 \mathrm{~kg} / \mathrm{m}^{2}\right)$ users $(P=0.15)$.

Conclusions: Our study demonstrated that if patients are relatively free to choose the insulin delivery method (minimal or no financial constraints), there were no differences in diabetes distress scores, HbA1c, or BMI between CSII and MDI. Therefore, people with T1DM may benefit from choosing the method of insulin delivery that will enable them to achieve individual goals and manage diabetes-related distress.
\end{abstract}

Keywords: Type 1 diabetes, Diabetes-related distress, Insulin delivery method, Continuous subcutaneous insulin infusion (CSII), Multiple daily injections (MDI).

\section{Introduction}

D IABETES REQUIRES A "complex, demanding, and often confusing set of self-care directives" in which "patients may become frustrated, angry, overwhelmed, and/or discouraged." 1 The American Diabetes Association (ADA) recommends psychosocial assessment as an integrated part of routine care for people with diabetes, which includes diabetes-related distress. ${ }^{2}$ Conceptually, diabetes-related distress captures people's perceptions about their diabetes self-management, support, emotional burden, and health care quality. ${ }^{1,3}$

\footnotetext{
${ }^{1}$ Diabetes Center of Excellence, Wilford Hall Medical Center, JBSA-Lackland Air Force Base, Texas.

${ }^{2}$ Division of Endocrinology, San Antonio Military Medical Center, JBSA-Ft. Sam Houston, Texas.

This material was presented as a poster at the 2019 American Diabetes Association Conference in San Francisco, CA.
} 
Diabetes-related distress can be assessed using the validated 17-item Diabetes-related Distress Scale (DDS-17), which measures diabetes-related distress in four distinct domains: (1) emotional burden (EB); (2) physician-related distress (PD); (3) regimen-related distress (RD); and (4) interpersonal distress (ID) (Table 1). ${ }^{1,3}$ In addition, there are three categories for DDS-17 scores: $<2.0=$ little or no distress; 2.0-2.9=moderate diabetes-related distress; and $\geq 3=$ high diabetes-related distress. ${ }^{1}$

Elevated diabetes-related distress is associated with poorer self-management, lower medication adherence, and poorer quality of life. ${ }^{4,5}$ Consequently, greater hemoglobin A1c (HbA1c) values correlate with higher diabetes-related distress, ${ }^{4-6}$ whereas lower diabetes-related distress is associated with increased self-efficacy and physician support. ${ }^{7}$

The relationship between diabetes-related distress and insulin delivery methods has not been well studied. The concept of satisfaction as it relates to insulin delivery modalities, however, has a more robust foundation in the literature. $\mathrm{Pa}$ -

\section{Table 1. The 17-Item Diabetes-Related} DisTRESS SCALE

\section{Emotional burden \\ 1. Feeling that diabetes is taking up too much of my} mental and physical energy every day

2. Feeling angry, scared, and/or depressed when I think about living with diabetes

3. Feeling that diabetes controls my life

4. Feeling that I will end up with serious long-term complications, no matter what I do

5. Feeling overwhelmed by the demands of living with diabetes

Physician-related distress

1. Feeling that my doctor doesn't know enough about diabetes and diabetes care

2. Feeling that my doctor doesn't give me clear enough directions on how to manage my diabetes

3. Feeling that my doctor doesn't take my concerns seriously enough

4. Feeling that I don't have a doctor who I can see regularly enough about my diabetes

Regimen-related distress

1. Feeling that I am not testing my blood sugars frequently enough

2. Feeling that I am often failing with my diabetes

3. Not feeling confident in my day-to-day ability to manage diabetes

4. Feeling that I am not sticking closely enough to a good meal plan

5. Not feeling motivated to keep up my diabetes selfmanagement

Interpersonal distress

1. Feeling that friends or family are not supportive enough of self-care efforts (e.g., planning activities that conflict with my schedule, encouraging me to eat the "wrong" foods)

2. Feeling that friends or family don't appreciate how difficult living with diabetes can be

3. Feeling that friends or family don't give me the emotional support that I would like

Responses are on a six-point continuum from $1=$ Not a problem; $2=\mathrm{A}$ slight problem; $3=\mathrm{A}$ moderate problem; $4=$ Somewhat serious problem; $5=\mathrm{A}$ serious problem; $6=\mathrm{A}$ very serious problem. tient satisfaction has been analyzed through surveys aimed at measuring treatment-related satisfaction, such as the Diabetes Treatment Satisfaction Questionnaire (DTSQ or DTSQc) and the Medical Outcome Study 36-Item ShortForm Survey (SF-36). An earlier study among people with type 1 diabetes mellitus (T1DM) with a history of poor glycemic management who were randomized to either continuous subcutaneous insulin infusion (CSII) or an intensive MDI (Aspart and NPH) regimen demonstrated a significant improvement in the subscales of "general health" and "mental health" in the CSII group, in addition to an improvement in HbAlc and glucose stability. ${ }^{8}$ However, there was no difference in treatment satisfaction by the DTSQ. ${ }^{8}$ More contemporary studies utilizing newer pump technology and longer acting basal insulin in the MDI groups, however, demonstrated higher satisfaction among CSII users compared with MDI. ${ }^{9,10}$ In addition to finding an improvement in satisfaction, ${ }^{10}$ lower rates of hypoglycemia and hyperglycemia was demonstrated among the CSII users compared with MDI, which likely affected treatment satisfaction. In a study by Hussain et al., ${ }^{9}$ the group that augmented their CSII with continuous glucose monitors (CGM) reported highest treatment satisfaction and health perception scores compared with MDI and CSII alone.

Because people with T1DM require comprehensive insulin therapy to manage blood glucose levels, we sought to explore the association of insulin delivery methods and diabetes distress in this group. Insulin delivery is achieved with a combination of basal and bolus (mealtime or prandial) dosing, which attempts to mimic normal pancreatic physiologic insulin release. Delivery methods include MDI through a syringe or pen injector or through CSII using a pump. Some evidence suggests that CSII improves treatment adherence, provides more accurate insulin dosing, and allows people to have increased flexibility in their lifestyle; however, this therapy can be expensive, requires a high level of engagement, and can be technically challenging. ${ }^{11}$ CSII is recommended for people with T1DM who prefer pump therapy over MDI and have been able to maintain healthy blood glucose levels. ${ }^{12,13}$ A systematic review and meta-analysis found CSII had modest improvements in $\mathrm{HbA} 1 \mathrm{c}^{14}$; however, there is no consensus regarding which insulin delivery therapy is best for people with T1DM. Furthermore, data are lacking about the association of diabetes-related distress with insulin delivery method. Thus, we explored the relationship of insulin delivery method (MDI vs. CSII) with diabetesrelated distress in patients with T1DM within multidisciplinary diabetes clinic.

\section{Methods}

Wilford Hall Ambulatory Surgical Center Institutional Review Board approval was obtained for this retrospective data analysis. Data were collected at the U.S. Air Force Diabetes Center of Excellence (DCOE) through chart reviews of clinical visits from June 2015 to August 2016. The DCOE is a Military Health System (MHS) diabetes specialty clinic, focusing on challenging cases of diabetes including people with T1DM.

Our population consists of all branches of active duty military members, retirees, and family members (beneficiaries of the MHS because of their relationship with the active 
duty member or retiree). Patients with T1DM were given a choice of insulin delivery method (MDI or CSII). Data concerning CGM use were not reliably recorded in the electronic record and individual use of CGM was not validated; therefore, this was not included in the analysis.

The DCOE began administering the DDS-17 in June 2015 as standard of care (Table 1). Responses for each item were on a six-point Likert scale from $1=$ Not a problem; $2=\mathrm{A}$ slight problem; $3=\mathrm{A}$ moderate problem; $4=$ Somewhat serious problem; $5=\mathrm{A}$ serious problem; $6=\mathrm{A}$ very serious problem. DDS-17 domains were calculated as a sum of the total and divided by the number of items in each domain: EB (five items); PD (four items); RD (five items); and ID (three items). It should be noted that total DDS-17 score should be avoided as it has less relevance than locating the source of distress; thus, discussion of the four individual domains will be presented. ${ }^{15}$

Inclusion criteria were adults (18 years and older) with T1DM receiving their diabetes care at the DCOE. As part of the regular clinical visit, all patients completed the DDS-17 with responses recorded by licensed vocational nurses. After input, the NoteWriter, an Excel-based clinical note writing platform created for use at the DCOE, calculated scores for total DDS-17 and each subscale consistent with established cut points of $<2.0$, little or no diabetes-related distress; 2.0 2.9, moderate diabetes-related distress; or $\geq 3.0$ as high diabetes-related distress. ${ }^{3}$ For analysis, the most recent DDS17 scores were used. In addition to the DDS-17, data included demographics (sex, age, ethnicity/race, and military status).

Data were analyzed using SPSS version 22 (IBM, Chicago, IL). Univariate analyses were conducted to characterize the sample according to group membership (CSII vs. MDI). Independent $t$-tests were conducted to assess differences in DDS-17 scores in the four domains for each group. Chisquare tests were conducted on each DDS-17 domain to determine if there were significant differences in distribution of level of diabetes-related distress (low, moderate, high) between groups.

\section{Results}

There were 203 people with T1DM who were seen at the DCOE and who completed the DDS-17 as part of standard care during the time period. People with T1DM also included those with diagnoses of pancreoprivic diabetes mellitus $(n=14)$ and latent autoimmune disease in adults $(n=43)$. People were categorized by method of insulin delivery as CSII (57.6\%) or MDI $(42.4 \%)$.

Although people can choose from a variety of CSII, most used a Medtronic pump (81.2\%). People on CSII were significantly more likely to be men $(53.1 \%)$, white $(64.2 \%)$, and a family member of an active duty service member $(63.0 \%)$ (Table 2). Significantly more women were on MDI (67.3\%) versus CSII (32.7\%). People using CSII had a diagnosis of diabetes for a significantly longer time than those on MDI (21.88 years; range, 19.0-78.0 years vs. 14.11 years; range, $<1-58.0$ years).

The four DDS-17 subscales were subjected to independent sample $t$-tests to examine differences between groups (CSII vs. MDI). Levene's test was conducted for all models, all models were significant; therefore, equal variances were not assumed. Normality checks were carried out and assumptions were met.

To determine if method of delivery influenced DDS-17 domain scores for people with T1DM, $t$-tests were conducted comparing DDS-17 subscales in people with T1DM and the method of delivery, CSII $(n=117)$ versus MDI $(n=86)$. Chisquare tests did not reveal significant differences in level of diabetes-related distress in any domain (Fig. 1). In addition, no significant differences were found in HbA1c $(P=0.22)$ between CSII $(7.9 \%$ or $63 \mathrm{mmol} / \mathrm{mol})$ and MDI $(8.1 \%$ or $65 \mathrm{mmol} / \mathrm{mol}$ ) and no significant differences were found in body mass index (BMI; $P=0.15)$ between people with T1DM using CSII $\left(M=28.33 \mathrm{~kg} / \mathrm{m}^{2}\right)$ and MDI $\left(28.49 \mathrm{~kg} / \mathrm{m}^{2}\right)$ (Table 2).

Further exploration of the level of distress within each insulin delivery method by DDS-17 domain (low, moderate, high) revealed no significant variation in each domain regarding CSII versus MDI (Fig. 2).

\section{Discussion}

In this study, we sought to better understand differences in diabetes distress levels in people with T1DM who used CSII versus MDI insulin delivery methods. Although one might consider advanced insulin-delivery technology to provide the ability to reduce distress in the T1DM population, we did not find this to be the case. Indeed, there were no differences in any of DDS-17 domains between these groups (Fig. 1). These findings suggest that neither method is better at assisting patients in reaching clinical goals or in decreasing diabetesrelated distress. Rather, the choice of insulin delivery method, with its associated potential for distress, is likely based on a variety of individual personal factors. On the one hand, CSII enables greater fine-tuning of insulin delivery with programmable basal rates and resources to calculate precise mealtime doses. Accordingly, this method may appeal to individuals who are technology savvy and enjoy tinkering with the latest devices. On the other hand, CSII requires a permanent connection of a device to the body, which may make individuals feel more encumbered compared with the MDI method. It would follow that individuals would naturally gravitate toward the method that provides less distress for them; of course, this assumes that each method is equally accessible.

We should also note that the vast majority of people in our study had low levels of distress in each of the DDS-17 domains (Fig. 2). Although each of the DDS-17 domains might be attributed to the insulin delivery method, RD is likely most relevant to our question. RD had very similar levels between the two groups with $>60 \%$ having low distress. We believe our findings support the notion that we should continue to have a variety of insulin delivery options for our population; people with T1DM may benefit from choosing the method of delivery to achieve individual goals.

It is not clear why women chose MDI over CSII at a significant rate in our population, whereas men were equally likely to use either of the insulin delivery methods. The older average age of our participants suggests that considerations regarding pregnancy and influence on preferred treatment modality were less likely, and recent reviews have suggested that glycemic control and maternal or neonatal outcomes are comparable in women choosing either delivery method. ${ }^{16}$ The decision to choose one mode of delivery over another 
Table 2. Sample Characteristics for Patients with Type 1 Diabetes Mellitus By Method of InSUlin Delivery

\begin{tabular}{lcccc}
\hline & Overall $(\mathrm{n}=203), \mathrm{n}(\%)$ & CSII $(n=117,57.6 \%)$ & $M D I(n=86,42.4 \%)$ & $\mathrm{P}$ \\
\hline Women & $107(52.7)$ & $35(32.7)$ & $72(67.3)$ & 0.003 \\
Men & $96(47.3)$ & $51(53.1)$ & $45(46.9)$ & $\mathrm{NS}$ \\
Mean age & $46.46(19-87)$ & 45.12 & 48.28 & 0.16 \\
Mean diabetes duration, years & 18.59 & 21.88 & 14.11 & $<0.001$ \\
Ethnicity/race & & & & \\
$\quad$ White & $134(66.0)$ & $86(64.2)$ & $48(35.8)$ & 0.008 \\
African American & $37(18.2)$ & $17(45.9)$ & $20(54.1)$ & NS \\
$\quad$ Hispanic/Latino & $24(11.8)$ & $12(50.0)$ & $12(50.0)$ & NS \\
$\quad$ Asian/Pacific Islander & $8(3.9)$ & $2(25.0)$ & $6(75.0)$ & NS \\
Military status & $16(7.9)$ & $7(43.8)$ & $9(56.3)$ & NS \\
$\quad$ Active duty & $52(25.6)$ & $25(48.1)$ & $27(51.9)$ & NS \\
$\quad$ Retirees & $135(66.5)$ & $85(63.0)$ & $50(37.0)$ & 0.03 \\
$\quad$ Family members & & & & \\
Clinical measures & $8.0 \%$ & $7.9 \%$ & $8.1 \%$ & 0.22 \\
$\quad$ HbA1c & 28.40 & 28.33 & 28.49 & 0.15 \\
BMI, kg/m & & & & \\
\hline
\end{tabular}

HbA1c and BMI were collected at the time of the DDS- 17 .

BMI, body mass index; CSII, continuous subcutaneous insulin infusion; DDS-17, 17-item Diabetes-related Distress Scale; MDI, multiple daily injections; NS, no significance; T1DM, type 1 diabetes mellitus.

involves provider-patient conversations about the relative pros and cons of each approach. It may be possible that there is an unconscious bias regarding the prescribing providers' perception of women's interest or comfort in using a technological solution to manage diabetes. ${ }^{17}$ Implicit bias is increasingly recognized as a driver of health care disparity in the treatment of various groups, including women. A recent International Data Corporation report ${ }^{18}$ found significant rates of gender bias in perceptions of women as candidates for the information technology jobs sector, and this suggests that there are widespread assumptions about women's interest in technology. Alternatively, CSII impact on body image may contribute to lower rates of CSII use by women. ${ }^{19}$ A review of influences on technology use and efficacy in T1DM found that women reported feeling more selfconscious about wearing their pump compared with men. ${ }^{19}$
Regardless of the insulin delivery method chosen, the mean $\mathrm{HbAlc}$ for the participants was statistically and clinically indistinct. ${ }^{20}$ This is consistent with the findings from a robust multicenter evaluation of the relative effectiveness of two approaches that found no difference in long-term $\mathrm{HbAlc}$, and additionally reported no differences in rates of hypoglycemia or psychological measures between treatment modalities. ${ }^{21}$ In addition, there was no statistically significant difference in BMI between those who used CSII and MDI, a finding consistent with those seen in the REPOSE trial. ${ }^{21}$

Although CSII has been found to improve $\mathrm{HbAlc}$ in people with type 2 diabetes mellitus compared with MDI, ${ }^{11,22}$ people with T1DM in our study using CSII did not have significantly different $\mathrm{HbA} 1 \mathrm{c}(7.9 \%$ or $63 \mathrm{mmol} / \mathrm{mol})$ than those using MDI $(8.1 \%$ or $65 \mathrm{mmol} / \mathrm{mol})$. Consistent with Maiorino et al., ${ }^{10}$ our study demonstrated no advantages to

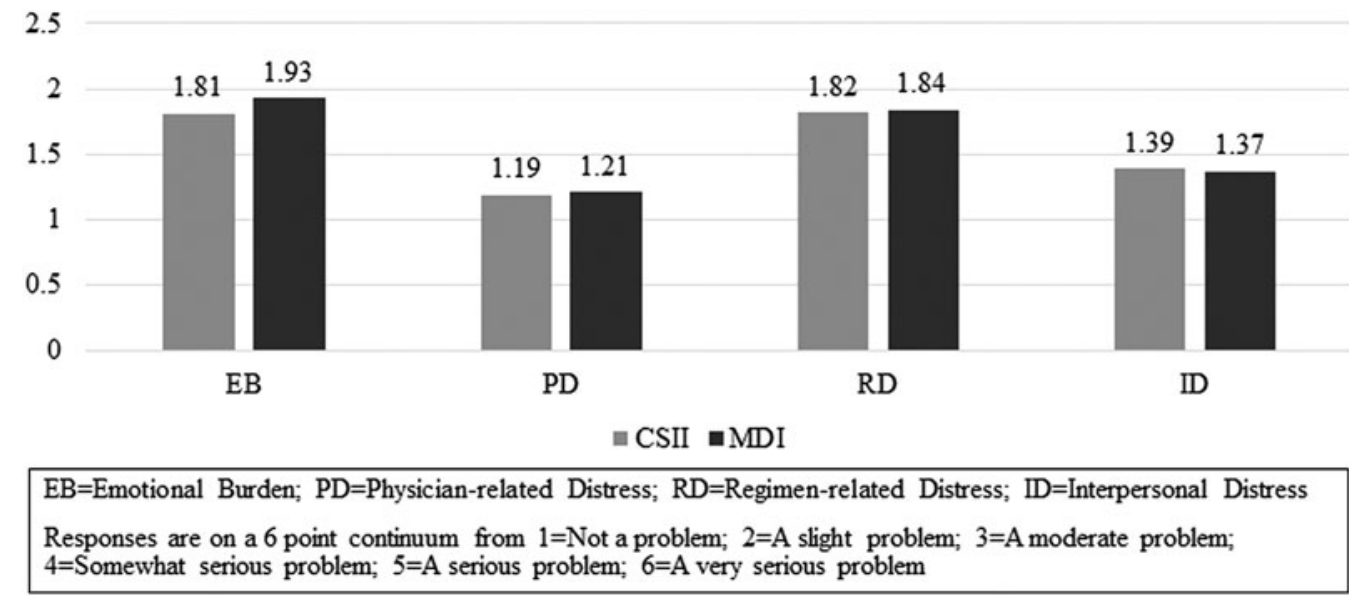

FIG. 1. Independent sample $t$-tests on DDS-17 domains by method of insulin delivery. DDS-17, 17-item Diabetes-related Distress Scale. 


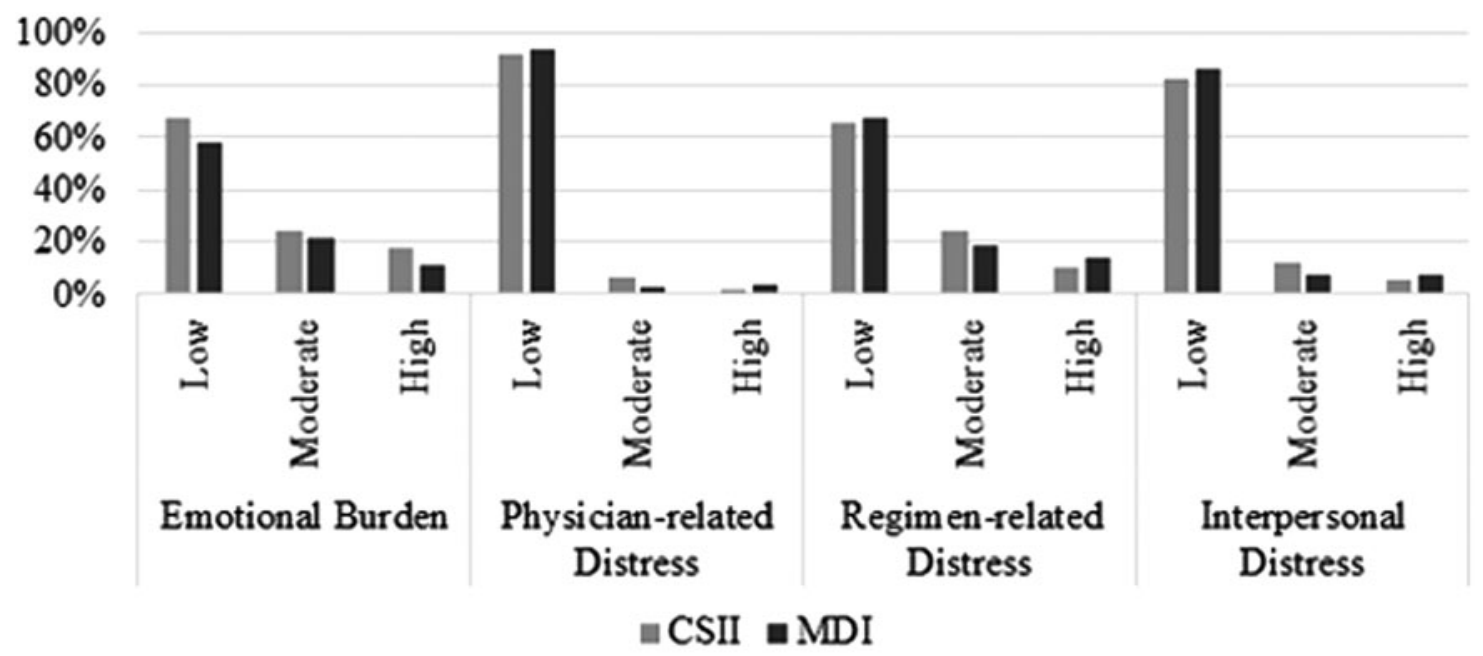

\section{Per Polonsky et al. $(2005)<2.0=$ Low diabetes-related distress; $2.0-2.9=$ Moderate diabetes-related distress; and $\geq 3=$ High diabetes-related distress}

FIG. 2. Level of distress by insulin delivery method and domain.

either CSII or MDI. DDS-17 was not statistically different between groups.

\section{Strengths and limitations}

Our study's main strength is one that is difficult to replicate outside the MHS. People on intensive insulin therapy within the MHS can choose between method of insulin delivery and type of CSII with little or no cost to the patient. When following the referral process, active duty patients and their dependents are not billed for CSII and supplies, whereas retirees and their dependents have a $20 \%$ copay on device and supplies. Thus, cost is usually not the primary determinant of method of insulin delivery. Even in nonmilitary environments, CSII was found to be cost-effective for people with T1DM who have poor glycemic management as CSII was associated with improved $\mathrm{HbA1c}$ and less hypoglycemia compared with MDI. ${ }^{23}$

Despite this strength, several limitations to our study exist. We did not analyze change in DDS-17 based on change of therapy (from MDI to CSII and/or CSII back to MDI) or change over time. The DDS-17 score is also not correlated with duration of currently chosen delivery method. Our population, while drawing from a wide array of backgrounds because of the transient nature of the military, was from one clinic, predominantly white, relatively well-managed $\mathrm{HbA} 1 \mathrm{c}$, and low diabetes-related distress across all domains. These data also may not be as applicable to current trends given the study was performed before the hybrid closed-loop technology being made available to our population.

Future research should include evaluation of DDS-17 before and after insulin delivery method changes, both MDI to CSII and CSII to MDI, along with monitoring of DDS-17 changes in each group over time. The duration a person has had diabetes or has used a specific insulin delivery method may also cause differences in DDS-17 scores, and correlation of DDS-17 with time of MDI and CSII therapy could provide beneficial information. Comparison between types of insulin pumps could provide insight into which CSII technology would provide best outcomes regarding DDS-17. Furthermore, evaluation of CSII users with CGMs versus those without CGMs could provide understanding to this specific technology and its association with DDS-17. Other investigations could potentially assess DDS-17 in people on MDI or CSII outside the MHS, where ease of obtaining your choice of delivery method is not as readily obtainable because of other substantiating factors, that is, out-of-pocket expenses.

Our study demonstrated that there were no differences in diabetes distress scores, HbA1c, or BMI between CSII and MDI when financial constraints were reduced or removed. As health care moves toward patient-centered care, people with T1DM may benefit from choosing the method of delivery that will enable them to achieve individual goals without increased diabetes-related distress, which may not always increase expense and/or be related to technology.

\section{Disclaimer}

The views expressed are those of the authors and do not reflect the official views or policy of the Department of Defense or its Components.

\section{Author Disclosure Statement}

No competing financial interests exist.

\section{Funding Information}

No funding was received for this article.

\section{References}

1. Polonsky WH, Fisher L, Earles J, et al.: Assessing psychosocial distress in diabetes: development of the diabetes distress scale. Diabetes Care 2005;28:626-631.

2. Young-Hyman D, De Groot M, Hill-Briggs F, et al.: Psychosocial care for people with diabetes: a position statement 
of the American Diabetes Association. Diabetes Care 2016; 39:2126-2140.

3. Fisher L, Hessler DM, Polonsky WH, Mullan J: When is diabetes distress clinically meaningful? Establishing cut points for the Diabetes Distress Scale. Diabetes Care 2012; 35:259-264.

4. Fisher L, Mullan JT, Arean P, et al.: Diabetes distress but not clinical depression or depressive symptoms is associated with glycemic control in both cross-sectional and longitudinal analyses. Diabetes Care 2010;33:23-28.

5. Schmitt A, Reimer A, Kulzer B, et al.: How to assess diabetes distress: comparison of the Problem Areas in Diabetes Scale (PAID) and the Diabetes Distress Scale (DDS). Diabet Med 2016;33:835-843.

6. Fisher L, Mullan JT, Skaff MM, et al.: Predicting diabetes distress in patients with type 2 diabetes: a longitudinal study. Diabet Med 2009;26:622-627.

7. Wardian JL, Sun F: Factors associated with diabetes-related distress: implications for diabetes self-management. Soc Work Health Care 2014;53:364-381.

8. DeVries JH, Snoek FJ, Kostense PJ, et al.: A randomized trial of continuous subcutaneous insulin infusion and intensive injection therapy in type 1 diabetes for patients with long-standing poor glycemic control. Diabetes Care 2002; 25:2074-2080.

9. Hussain T, Akle M, Nagelkerke N, Deeb A: Comparative study on treatment satisfaction and health perception in children and adolescents with type 1 diabetes mellitus on multiple daily injection of insulin, insulin pump and sensoraugmented pump therapy. SAGE Open Med 2017;5: 2050312117694938.

10. Maiorino MI, Bellastella G, Casciano O, et al.: The effects of subcutaneous insulin infusion versus multiple insulin injections on glucose variability in young adults with type 1 diabetes: the 2-year follow-up of the observational METRO study. Diabetes Technol Ther 2018;20:117-126.

11. Pickup JC, Reznik Y, Sutton AJ: Glycemic control during continuous subcutaneous insulin infusion versus multiple daily insulin injections in type 2 diabetes: individual patient data meta-analysis and meta-regression of randomized controlled trials. Diabetes Care 2017;40:715-722.

12. American Diabetes Association: 7. Diabetes Technology: Standards of Medical Care in Diabetes-2019. Diabetes Care 2019;42(Suppl 1):S71-S80.

13. Grunberger G, Bailey T, Cohen A, et al.: Statement by the American Association of Clinical Endocrinologists Consensus Panel on insulin pump management. Endocr Pract 2010;16:746-762.

14. Yeh HC, Brown TT, Maruthur N, et al.: Comparative effectiveness and safety of methods of insulin delivery and glucose monitoring for diabetes mellitus: a systematic review and meta-analysis. Ann Intern Med 2012;157:336347.

15. Fenwick EK, Rees G, Holmes-Truscott E, et al.: What is the best measure for assessing diabetes distress? A comparison of the Problem Areas in Diabetes and Diabetes Distress Scale: results from Diabetes MILES-Australia. J Health Psychol 2018;23:667-680.

16. Umpierrez GE, Klonoff DC: Diabetes technology update: use of insulin pumps and continuous glucose monitoring in the hospital. Diabetes Care 20181;41:1579-1589.

17. FitzGerald C, Hurst S: Implicit bias in healthcare professionals: a systematic review. BMC Med Ethics 2017;18:19.

18. International Data Corporation (IDC): 2018 women in tech: unconscious bias, parity, and the path forward. 2018. https:// www.witi.com/IDC-2018-Women-in-Tech.pdf views on 18 June 2019.

19. Franklin V: Influences on technology use and efficacy in type 1 diabetes. J Diabetes Sci Technol 2016;10:647-655.

20. Sacks DB, Arnold M, Bakris GL: Evidence-Based Laboratory Medicine Committee of the American Association for Clinical Chemistry. Guidelines and recommendations for laboratory analysis in the diagnosis and management of diabetes mellitus. Diabetes Care 2011;34:e61-e99.

21. REPOSE Study Group: Relative effectiveness of insulin pump treatment over multiple daily injections and structured education during flexible intensive insulin treatment for type 1 diabetes: cluster randomised trial (REPOSE). BMJ 2017;356:j1285.

22. Vigersky RA, Huang S, Cordero TL, et al.: Improved HbA1c, total daily insulin dose, and treatment satisfaction with insulin pump therapy compared to multiple daily insulin injections in patients with type 2 diabetes irrespective of baseline c-peptide levels. Endocr Pract 2018;24:446452.

23. Roze S, Smith-Palmer J, Valentine W, et al.: Costeffectiveness of continuous subcutaneous insulin infusion versus multiple daily injections of insulin in Type 1 diabetes: a systematic review. Diabet Med 2015;32:14151424.

Address correspondence to: Jana L. Wardian, PhD Diabetes Center of Excellence Wilford Hall Medical Center 1100 Wilford Hall Loop JBSA-Lackland Air Force Base, TX 78236-5300

E-mail: jana.1.wardian.ctr@mail.mil 